\title{
Frühinterventionen bei Suchtgefährdung - Ein Überblick
}

\author{
E. Rühling \\ M. Stich \\ C. Hartwig
}

\author{
Early Interventions for Drug Users at Risk of Becoming Addicted - An Overview
}

\section{Zusammenfassung}

Menschen mit einem schädlichen Drogenkonsum werden vom Sucht- und Drogenhilfesystem in der Regel sehr spät erreicht. Es ist aus der Praxis bekannt, dass bei Substanzmissbrauch professionelle Hilfe oft erst nach Jahren aufgesucht wird, zu einem Zeitpunkt, wo bereits massive Folgeprobleme bestehen und hohe Folgekosten für die Gesellschaft entstanden sind.

Die frühzeitigen Interventionsstrategien gehören grundsätzlich in den Bereich der zielgruppenspezifischen Sekundärprävention, obwohl eine strikte Abgrenzung zu primärpräventiven Maßnahmen nicht immer eindeutig möglich ist. Frühinterventionen sollen sich an Personen richten, die ein erhöhtes Abhängigkeitsrisiko zeigen, oder an Personen, die bereits ein manifestes Risikoverhalten - jedoch ohne Abhängigkeitsdiagnose nach ICD-10 - aufweisen.

In dieser Arbeit werden beispielhaft deutschsprachige Frühinterventionsprojekte dargestellt, die sich a) direkt an Betroffene oder b) an Personal bzw. Therapeuten wenden, um diese in Frühinterventionsstrategien auszubilden, und c) an Internet-User. Vor diesem Hintergrund werden Möglichkeiten einer Ausweitung früher Interventionen diskutiert.

\section{Schlüisselwörter}

Frühinterventionen - Kurzinterventionen - motivierende Gesprächsführung $\cdot$ Sekundärprävention

\section{Abstract}

People with harmful drug consumption are usually reached very late by addiction and drug services. Experience shows that professional help is often sought only after years of drug abuse, when severe consequences already exist resulting in high subsequent costs for the community.

Early intervention strategies are basically a part of secondary prevention aimed at specific target groups, though a clear delineation between primary prevention and early intervention is not always possible. Early interventions should aim at persons at an increased risk of becoming addicted or at persons already displaying risk behaviour - though without an addiction diagnosis according to ICD-10.

In this essay early intervention projects of German language will be exemplarily presented, which address a) persons directly concerned, b) personnel and therapists to train them in early intervention strategies, and c) internet users. Based on this background, possibilities of expanding early interventions are discussed.

Key words

Early interventions $\cdot$ short interventions $\cdot$ motivational interviewing · secondary prevention 
Einleitung

Personen mit schädlichem Drogenkonsum werden vom Suchtund Drogenhilfesystem in der Regel sehr spät erreicht, professionelle Hilfe nehmen sie häufig erst nach Jahren des Suchtmittelmissbrauchs in Anspruch. Aus diesem Grund befindet sich, gemessen an der hohen Zahl von Risikokonsumenten und Alkoholmissbrauchenden, nur ein sehr kleiner Teil in adäquater Behandlung. Im Aktionsplan Drogen und Sucht von November 2003 wird die Zahl der Menschen mit gravierenden Alkoholproblemen auf über 9 Millionen geschätzt, wovon etwa 1,6 Millionen eine Alkoholabhängigkeit aufweisen [1]. Das bestehende Suchtkrankenversorgungssystem erreicht jedoch nur einen Bruchteil der Betroffenen. Eine repräsentative Bevölkerungsuntersuchung in der Region Lübeck hat beispielsweise ergeben, dass $71 \%$ der Alkoholabhängigen keinen Kontakt zum vorhandenen Suchthilfesystem haben. Lediglich 14,5\% nehmen Angebote der Suchtkrankenversorgung z. B. im Rahmen von Entwöhnungsbehandlungen in Anspruch [2]. Für Risikokonsumenten und Alkoholmissbraucher, also Personen, die noch keine manifeste Abhängigkeit aufweisen, bestehen nahezu keine entsprechenden Hilfsangebote [2].

In den letzten Jahren hat sich bestätigt, dass besonders durch gezielte Interventionen in frühen Stadien der Suchtkarriere eine Umkehr eingeleitet werden kann. Denn riskantes Trinkverhalten mündet nicht per se in Alkoholmissbrauch, genauso wie Substanzmissbrauch nicht in jedem Fall eine Abhängigkeit zur Folge hat, wenn das Verhalten rechtzeitig erkannt und behandelt wird bzw. die Vermittlung weitergehender Hilfe erfolgt [3]. Frühintervention wird deshalb als mögliche Herangehensweise bei Substanzmittelmissbrauch eine immer bedeutendere Rolle zugesprochen. Es ist davon auszugehen, dass - auch wenn sich die meisten Studien und Projekte der Frühinterventionen bisher an Personen mit riskantem Alkoholkonsum richten - eine Übertragbarkeit auf Projekte, die sich auf den Missbrauch anderer psychoaktiver Substanzen konzentrieren, möglich ist.

\section{Begriffserklärung}

In der gängigen Literatur findet sich oftmals eine Darstellung der Frühintervention ähnlich der des gesundheitswissenschaftlichen Verständnises der Sekundärprävention. Diese hat zum Ziel, Erkrankungen und deren Risikofaktoren möglichst frühzeitig zu erkennen und zu therapieren bzw. zu beseitigen. In diesem Sinne wenden sich Frühinterventionen zielgruppenorientiert an Personen, die ein missbräuchliches oder riskantes Konsumverhalten aufweisen. Frühinterventionenen versuchen, Personen mit moderaten bis starken Problemen (ohne dass bereits eine deutliche Abhängigkeit nach ICD zu diagnostizieren wäre) zu helfen, ihren Substanzkonsum zu verringern, zu kontrollieren oder ganz einzustellen. Die Mehrzahl der Frühinterventionsprojekte richtet sich an Jugendliche oder junge Erwachsene, es ist aber durchaus auch eine Orientierung auf „ältere“ Zielgruppen möglich.

Auf den ersten Blick unterscheiden sich Frühinterventionen mit ihrer zielgruppenspezifischen Herangehensweise von primärpräventiven Maßnahmen, die als allgemeine Prophylaxe in erster Linie darum bemüht sind, Gesundheitsstörungen oder Erkran- kungen an unselektierten Personengruppen zu vermeiden (z.B. im Rahmen von Aufklärungskampagnen). Primärprävention soll jedoch nicht nur zur generellen Vermeidung des Drogenkonsums beitragen, sondern zielt auch darauf ab, den Übergang vom Probierstadium zum regelmäßigen Konsum zu vermeiden.

Eine klare Abgrenzung zwischen den einzelnen Stufen der Prävention und eine entsprechend exakte Einordnung des Begriffes der Frühintervention ist deshalb letztlich nicht möglich. Hingegen sind viele Projektansätze der Frühinterventionen zwar vorrangig im Bereich der zielgruppenspezifischen Sekundärprävention einzuordnen, setzen aber auch auf eine Beteiligung der primären Präventionsansätze. Dieser Tendenz entspricht eine Empfehlung, die die Drogen- und Suchtkommission der Bundesregierung in ihrem Abschlussbericht im Juni 2002 zur Verbesserung der Suchtprävention erarbeitet hat. Sie besagt unter anderem, dass die Trennung von primär- und sekundärpräventiven Maßnahmen zugunsten eines übergreifenden Ansatzes aufgegeben werden sollte, der sich stärker an Entwicklungsprozessen im Kontext von Lebenswelten orientiert.

Es erscheint sinnvoll, zu Beginn dieses Artikels eine eigenständige Begriffserklärung voranzustellen:

Frühinterventionen richten sich an

- Personen mit erhöhtem Abhängigkeitsrisiko (z. B. Kinder von alkoholabhängigen Eltern oder Kinder mit sexuellen Missbrauchserfahrungen) und

- Personen, die bereits ein manifestes Risikoverhalten zeigen, ohne nach den diagnostischen Kriterien von ICD 10 oder DSMV eine Abhängigkeit aufzuweisen (z. B. Personen, die am Wochenende „binge-drinking“ praktizieren).

\section{Gesundheitsökonomische Aspekte}

Suchtmittelmissbrauch oder Substanzmittelabhängigkeit hat für die Betroffenen nicht nur Probleme und Leid zur Folge, sondern stellt auch einen großen Kostenfaktor im Gesundheitswesen dar.

Im Jahr 2000 starben weltweit etwa 7 Millionen Menschen an den Folgen von Alkohol-, Tabak- und illegalem Drogenkonsum [4], wobei die legalen Suchtmittel Alkohol und Tabak dabei den Hauptanteil ausmachten (zum Vergleich: Es sterben jährlich etwa 1,2 Millionen Menschen im Straßenverkehr [5]). Neben den hohen Mortalitätsraten sind die gravierenden Krankheitsfolgen zu nennen, die mit hohen gesellschaftlichen Kosten verbunden sind. Nach einer WHO-Schätzung werden weltweit 6\% des Bruttosozialproduktes einer Industrienation für alkoholassoziierte Folgeschäden aufgewendet [6]. Allein die Kosten der alkoholbezogenen Erkrankungen werden in Deutschland pro Jahr auf etwa 20 Milliarden Euro geschätzt [7].

Diese enormen Folgekosten sind jedoch nicht ausschließlich auf den abhängigen, sondern auch auf den „nichtsüchtigen“ Gebrauch zurückzuführen, d.h. auf Personen, die nach ICD oder DSM nicht die Kriterien für eine Abhängigkeits- oder Missbrauchsdiagnose erfüllen [8]. Alkoholbedingte Verkehrs- und sonstige Unfälle werden beispielsweise mehrheitlich durch Personen verursacht, die zwar situativ zu viel getrunken haben, aber 
nicht abhängig sind [8]. Aus gesundheitsökonomischer Sicht ist es daher ratsam, nicht erst gegen die Folgekrankheiten zu intervenieren, sondern viel früher und insgesamt gezielter dem Drogenkonsum zu begegnen. „Eine Politik der Frühintervention und Prävention dürfte langfristig die beste Kostenreduktionspolitik darstellen“ [9].

Im niedergelassenen Bereich sind im Leistungskatalog noch keine Abrechnungsziffern für Frühinterventionen vorgesehen, durch die Einführung entsprechender Abrechnungsmöglichkeiten können Anreize, frühzeitig zu intervenieren, geschaffen werden. Neben der Verbesserung der Rahmenbedingungen für eine frühzeitige, methodische Reaktion auf Substanzmittelmissbrauch ist darüber hinaus auch die Entwicklung eines funktionierenden Behandlungsnetzes notwendig. Eine Vernetzung der verschiedenen Akteure des Suchthilfesystems, wie Sozialarbeiter in den Drogeneinrichtungen, Suchtschwerpunktpraxen, Drogenbeauftragte der Schulen und Betriebe sowie Selbsthilfegruppen etc., ist eine erforderliche strukturelle Voraussetzung, um die Versorgung von Personen mit missbräuchlichem Substanzkonsum oder von Personen einer Hochrisikogruppe zu optimieren.

\section{Handlungsfelder}

Frühinterventionen erschließen neue Zugangswege und Aktionsfelder im Präventionsbereich (Tab.1). Sie leisten damit einen Beitrag, Personen mit riskantem Substanzkonsum, die bisher nicht vom Suchthilfesystem erfasst wurden, frühzeitig zu erreichen.

Tab. 1 Einsatzorte von Frühinterventionen

\begin{tabular}{|ll|}
\hline Hausarzt/Facharzt & Internet \\
\hline Allgemeinkrankenhaus & Arbeitsplatz \\
\hline Schule/Berufsschule & Arbeitsamt \\
\hline Apotheke & Jugendzentren \\
\hline Jugendhilfeeinrichtungen & Krankenkassen \\
\hline
\end{tabular}

Suchtkranke und Suchtgefährdete werden zumeist in Arztpraxen, im Krankenhaus, am Arbeitsplatz, in der Schule oder in Jugendeinrichtungen auffällig. Diese Einrichtungen eignen sich deshalb besonders dazu, suchtspezifische Frühinterventionen durchzuführen oder eine Vermittlung in entsprechende Einrichtungen zu leisten. Darüber hinaus können über das Medium Internet Kontakte hergestellt werden und Maßnahmen der Frühinterventionen stattfinden.

Im folgenden Abschnitt soll auf bestimmte Handlungsfelder der Frühintervention unter Berücksichtigung ihrer Vorteile näher eingegangen werden.

\section{Hausarzt}

Laut des Drogen- und Suchtberichtes der Bundesregierung aus dem Jahre 2003 ist der quantitativ bedeutsamste Bereich für die Versorgung von Menschen mit Alkoholproblemen die Hausarztpraxis. Etwa 70 bis $80 \%$ der Menschen mit Alkoholproblemen gehen mindestens einmal im Jahr zu ihrem Hausarzt $[3,10]$. Diese
Zahlen signalisieren Reserven in der Erreichbarkeit von Menschen mit Substanzmittelmissbrauch vor allem im niedergelassenen Versorgungssystem und verstärken das enorme Handlungspotenzial der frühzeitigen Erkennung und Behandlung.

Für die Beratung der Patienten in der Hausarztpraxis spricht neben der guten Erreichbarkeit die Rolle des Hausarztes als Vertrauensperson, die sich durch den meist schon jahrelangen Kontakt entwickelt hat. Speziell bei der Alkoholproblematik ist eine Vielzahl von Erkrankungen direkt oder indirekt mit dem Alkoholkonsum verbunden und bietet dadurch günstige Anknüpfungspunkte für das beratende Gespräch [2]. Die Bundeszentrale für gesundheitliche Aufklärung (BZgA) hat in Zusammenarbeit mit der Bundesärztekammer, dem Fachverband Sucht e.V. und der Deutschen Hauptstelle gegen die Suchtgefahren im Jahre 2001 den Beratungsleitfaden „Kurzintervention bei Patienten mit Alkoholproblemen“ herausgegeben, der sich primär an niedergelassene Ärzte wendet. Der Leitfaden enthält praxisorientierte Leitlinien für die Diagnostik und Behandlung von Patienten mit riskantem, schädlichem Alkoholkonsum und Alkoholabhängigkeit [11]. Ein zusätzlicher Schritt in Richtung effizienter Behandlung von Abhängigen ist - gerade im Falle einer weiterführenden Behandlung - die Förderung der intensiven Zusammenarbeit zwischen dem spezifischen Suchthilfesystem und den niedergelassenen Ärzten [12].

\section{Krankenhaus}

Frühinterventionen im Allgemeinkrankenhaus führen zu einer deutlichen Steigerung der Inanspruchnahme weiterführender Hilfen (z.B. Suchtberatungsstellen) bei Alkoholabhängigen [13]. Krankenhausbezogene Frühinterventionen eignen sich deshalb sehr gut, weil dort die Änderungsbereitschaft der Patienten aufgrund der Auseinandersetzung mit der Erkrankung (Einweisungsgrund) erhöht ist. Weiter können sich die Gegebenheiten der stationären Behandlung (zeitweilige Abstinenz, körperlicher Entzug) günstig auf eine Beratung und weiterführende Maßnahmen auswirken [2].

\section{Schule}

Durch die allgemeine Schulpflicht ist über die Schule eine hohe Erreichbarkeit von Kindern und Jugendlichen garantiert. Interventionen, die didaktisch und methodisch gut aufbereitet sind, können in den Unterricht und damit in einen bestehenden Klassenverband integriert werden.

Schulprojekte richten sich häufig zuerst an die gesamte Schulklasse mit der Absicht, die eigentliche Zielgruppe (z.B. Raucher) anzusprechen und zu motivieren, an spezifischen Maßnahmen (z.B. speziellen Arbeitskreisen für rauchende Schüler) teilzunehmen. Darüber hinaus nimmt der Vertrauenslehrer im Bereich der Frühintervention eine entscheidende Rolle ein. Entweder kann er weiterführende Hilfe vermitteln oder selber die Methoden der Frühinterventionen anwenden, ohne dass Schulnoten bedeutend sind.

\section{Internet}

Es gibt inzwischen einige Frühinterventionen in Form von Internetauftritten. Chaträume haben den Vorteil der Anonymität und Betroffene können sich mit "Gleichgesinnten“ austauschen. Außerdem kann virtuell professionelle Hilfe in Anspruch genommen werden, die aus der Distanz ganz unverbindlich berät, 
Orientierung bietet und ermutigt, Hilfsangebote frühzeitig in Anspruch zu nehmen.

\section{Prozessablauf}

Generell gibt es zwei Phasen im Frühinterventionsprozess: die Identifizierung der Personen und ihrer Probleme sowie die Bereitstellung der unterschiedlichen Interventionen. Im Folgenden wird der erste Schritt der Frühintervention, das Screening, dargestellt:

\section{Screening}

Zur frühzeitigen Erkennung gesundheitsschädlicher Entwicklungen, bedingt durch den Konsum psychoaktiver Substanzen, dienen - zusätzlich zu den leicht erkennbaren klinischen Merkmalen - diagnostische Instrumentarien. Neben Laborparametern können Screening-Fragebögen, die auf Selbstaussagen der Patienten zurückgreifen, die Entdeckungsrate von Alkoholabhängigkeit deutlich verbessern. Das Fragebogenverfahren hat sich in der Praxis als sehr effektiv und effizient erwiesen. Einige der gängigen Instrumentarien sollen hier exemplarisch genannt werden:

- LAST (Lübecker Alkoholabhängigkeits- und -missbrauchsScreening-Test), [14] effizientes Verfahren in nur sieben Fragen;

- CAGE, ein einfaches Screening mit vier Fragen zur Selbstbeurteilung (Cut down on drinking? Annoyed by complaints about drinking? Guilty about drinking? Had an Eye-opener first thing in the morning?) [15], einfaches Screening mit vier Fragen zur Selbstbeurteilung;

- MALT (Münchner Alkoholismus-Test) [16], Kombination von Selbst- und Fremdbeurteilung;

- AUDIT (Alcohol Use Disorders Identification Test) [17] aufwändigeres Verfahren;

- BASIC (Brief Alcohol Screening Instrument for Primary Care) [2].

Die Diagnoseverfahren LAST, MALT, AUDIT und BASIC hinterfragen speziell die Alkoholabhängigkeit. Bei CAGE ist eine Modifizierung möglich, die auch den Konsum anderer Substanzen screent.

\section{Methoden}

Der zweite Schritt der Frühinterventionen besteht aus der eigentlichen Intervention. Methoden der Frühintervention, die zumeist in Form von Kurzinterventionen durchgeführt werden, sollen eine Veränderungsbereitschaft und Behandlungsmotivation in frühen Phasen der Abhängigkeitsentwicklung fördern.

Im deutschsprachigen Raum sind die Maßnahmen der Kurzintervention nicht einheitlich festgelegt. Es gibt unterschiedliche Auslegungen der Dauer und der Frequenz der Interventionen. In der Regel aber finden Kurzinterventionen bis zu viermal statt mit einem Zeitumfang von jeweils 5 bis 60 Minuten [18].

Miller und Sanchez beschreiben unter dem Akronym FRAMES die wesentlichen Elemente der Kurzinterventionen [19]:

- Feedback (Rückmeldung bezüglich negativer Folgen geben);

- Responsibility (helfen Verantwortung für das eigene Verhalten zu übernehmen);

- Advice (Beratung hinsichtlich der Ziele und Vorgehensweisen);
- Menu (Wahlmöglichkeiten zwischen verschiedenen Veränderungsalternativen);

- Empathy (Empathie, einfühlendes Verstehen);

- Self-Efficacy (Förderung der Selbstwirksamkeitserwartungen bezüglich Veränderungen).

Aufgabe des Behandelnden ist es zu erkennen, welche Art der Motivationsbildung bei dem jeweiligen Individuum angebracht ist. Konfrontatives Vorgehen bei der Beratung von Personen mit Konsumproblemen ist zu vermeiden, da auf diesem Wege lediglich Widerstand bei ihnen erzeugt wird.

Nachfolgend werden exemplarisch das Transtheoretische Modell sowie ein möglicher Ansatz der Kurzintervention, das so genannte „Motivational Interviewing“, dargestellt.

\section{Das Transtheoretische Modell}

Das Transtheoretische Modell (TTM) ist ein Modell der Verhaltensänderung („Behavior Change“). Es ist ein integratives Modell, das Elemente aus anderen Theorien beinhaltet (z. B. Selbstwirksamkeit von Bandura). Mit dem TTM wird die Bereitschaft zu einer Einstellungs- und Verhaltensänderung in Hinblick auf ein konkretes definiertes Problemverhalten beschrieben. Das Hauptmerkmal des TTM sind die fünf bzw. sechs Veränderungsphasen („Stages of Change“ [SoC]). Sie beschreiben die zeitliche Dimension sowie abhängige und unabhängige Variablen. $\mathrm{Zu}$ den abhängigen Variablen („Dependent Measures“) gehören Entscheidungsbalance („Decisional Balance“) und Selbstwirksamkeit („Self-Efficacy“). Die Entwicklungsprozesse („Processes of Change“) werden den unabhängigen Variablen („Independent Measures“) zugeordnet [20, 21].

\section{Die Stages of Change}

Prochaska und DiClemente beschreiben sechs „Stages of Change“ (SoC), wobei die sechste Phase erst später hinzugefügt wurde.

1. Phase der Absichtslosigkeit (Precontemplation)

In diesem Stadium der Absichtslosigkeit befinden sich die Menschen, die nicht in den nächsten sechs Monaten planen, ihr problematisches Verhalten zu ändern. Die Gründe für diese Haltung liegen in fehlender Motivation, mangelnder Information und vorangegangenen (häufigen) Misserfolgserfahrungen. Die Individuen vermeiden das Ansprechen der Problematik, sie reflektieren nicht das gesundheitsschädliche Verhalten.

2. Phase der Absichtsbildung (Contemplation)

Menschen in diesem Stadium planen eine Verhaltensänderung. Die Vor -und Nachteile der angedachten Veränderung werden abgewogen. Ambivalentes Verhalten führt häufig zu einem langen Verbleiben in dieser Phase der Absichtsbildung und reduziert die Möglichkeiten der Vermittlung in traditionelle Programme.

3. Phase der Vorbereitung (Preparation) Menschen in dieser Phase der Vorbereitung haben die Intention, in unmittelbarer Zukunft (in weniger als einem Monat) ihr Verhalten zu verändern. Sie haben sich grundlegend informiert und können sich selbst gut einschätzen. Dieser Zeitpunkt eignet sich, Programme, die einen deutlichen Handlungscharakter haben, zu implementieren. 
4. Phase der aktiven Verhaltensänderung (Action)

In dieser Phase wird die angestrebte Verhaltensänderung (Rauchen beenden, Alkoholkonsum einstellen) vollzogen. Die Umwandlung des neu erlernten Verhaltens in die Alltagsroutine erfordert viel Energie und Zeit. Die Sensibilität wird in dieser Phase der aktiven Verhaltensänderung, die sechs Monate umfasst, als groß beschrieben.

5. Phase der Aufrechterhaltung (Maintenance)

Während der Phase der Aufrechterhaltung wird die Verhaltensänderung zur Routine. Das Hauptaugenmerk liegt auf der Rückfallvermeidung. Diese Phase kann zwischen sechs Monaten und fünf Jahren dauern.

6. Phase der Stabilisierung (Termination)

In dieser letzten Phase ist das Problem der Rückfallgefährdung nicht mehr vorhanden. Die Person vertraut den erlernten Fähigkeiten und Unsicherheiten liegen nicht mehr vor.

Das Transtheoretische Modell findet Anwendung in der öffentlichen Gesundheitsvorsorge, klinischen Psychologie, Gesundheitspsychologie und in der primären, sekundären und tertiären Prävention. Ursprünglich wurde das TTM zur Raucherentwöhnung entwickelt. Auch bei anderem Suchtverhalten wie Alkoholmissbrauch, Kokain- und Heroinabhängigkeit, Esssucht sowie missbräuchlichem Spielverhalten kommt es zum Einsatz. Des Weiteren wird es zur HIV-Prävention, beim Brustkrebsscreening, zur Prävention von Hautkrebs und bei der Durchführung von Diäten angewendet [22].

\section{Motivational Interviewing}

Motivational Interviewing (MI), die motivierende Gesprächsführung, ist ein sowohl klientenzentrierter als auch direkter Ansatz der Gesprächsführung zur Erhöhung der Eigenmotivation von Menschen, ein problematisches Verhalten (z. B. Suchtmittelabusus) zu ändern. Der von Miller und Rollnick stammende Ansatz ist mittlerweile international besonders in der Suchtbehandlung weit verbreitet $[23,24]$.

Der Ansatz des MI geht davon aus, dass Ambivalenz den Menschen in vielen wichtigen Lebensentscheidungen begleitet. Gerade im Zusammenhang mit der Suchtproblematik ist das ambivalente Verhalten, also die begründete innere Zwiespältigkeit, ein zentrales Phänomen. Miller und Rollnick erklären den Konflikt dieses Wechselspiels der Abwägungen zwischen Vor- und Nachteilen einer Verhaltensänderung bzw. einer Nichtänderung mit dem Bild einer Waage mit zwei Waagschalen [25]. Durch diese Auseinandersetzung zwischen Weitermachen und Aufhören kommt es in den meisten Fällen zu einer differenzierten Sichtweise gegenüber dem Drogenkonsum, die wiederum die Basis einer stabilen Veränderungsmotivation und einer individuell abgestimmten, differenzierten Zielfindung darstellt. Die Anerkennung von Ambivalenzen gegenüber einer Änderung des Suchtverhaltens ist ein erster Schritt im motivierenden Umgang mit Suchtmittelproblemen [18]. Die vier Grundprinzipien des MI beinhalten folgende Elemente:

- Empathie ausdrücken („Express Empathy“)

Empathie beinhaltet die Fähigkeit und Bereitschaft, Einstellungen und Gefühle aus Sicht des Klienten zu betrachten, ohne sie zu bewerten oder zu kritisieren. Das respektvolle Betrachten und Zuhören ist nicht gleichzusetzen mit einem Zu- billigen des Verhaltens. Ambivalente Einstellungen sollen lediglich zugelassen werden. Fühlen sich Klienten verstanden und angenommen, sind sie für eine Verhaltensänderung leichter zu motivieren. Empathie wird als das wesentliche Charakteristikum der motivierenden Gesprächsführung angesehen.

- Diskrepanzen entwickeln („Develop Discrepancy“)

Eine Veränderungsmotivation entwickelt sich, wenn Personen eine Diskrepanz zwischen der gegenwärtigen Situation und zukünftigen Zielen wahrnehmen. MI-Therapeuten sollen Klienten deshalb dazu bringen, Widersprüche in ihrer Selbstwahrnehmung zu entdecken und sich bewusst zu machen.

- Den Widerstand aufnehmen („Roll with Resistance“)

„Gehe mit dem Widerstand, anstatt dich gegen ihn zu stellen“ [24]. Widerstandsverhalten bei Klienten (z. B. Suchtprobleme zu leugnen oder die Kompetenz des Therapeuten in Abrede zu stellen) wird begünstigt, wenn man einen Menschen gegen seinen Willen überzeugen möchte, sich zu ändern. Daher sollte ein geschulter MI-Therapeut konstruktiv mit Klientenwiderstand umgehen und erste Anzeichen zum Anlass nehmen, das eigene Vorgehen und sein Gesprächsverhalten zu überdenken. Dies wird dem Therapeuten leichter fallen, je mehr er Klientenwiderstand wertschätzen und als normales, verstehbares Verhalten auffassen kann [24].

- Selbstwirksamkeit, Veränderungszuversicht fördern („Support Self-Efficacy“)

Selbstwirksamkeit fördern heißt, den Glauben des Klienten an sich selbst, sein Selbstbewusstsein zu stärken und sich selbst als jemanden wahrzunehmen, der mit einer bestimmten Aufgabe fertig wird. Aufgabe von Beratern ist es, sich gerade nicht auf die Schwächen und Defizite zu konzentrieren, sondern die Fähigkeiten, Stärken und (sozialen) Ressourcen der Betroffenen in den Mittelpunkt zu stellen [24]. Gemeinsam wird ein realistischer Schritt in Richtung einer Verhaltensänderung gefunden. Die Zielfindung ist ein zentraler Baustein der Selbstwirksamkeitsüberzeugung.

Die nachfolgend aufgelisteten Methoden dienen der Umsetzung der vier MI-Grundprinzipien [25]:

- offene Fragen („Open Questions“);

- aktives Zuhören („Reflective Listening“);

- Würdigung bzw. Wertschätzung von Verhaltensweisen („Affirmation“);

- Methoden zur Förderung veränderungsbezogener Äußerungen von Klienten („Change Talk“). Bei diesen Methoden wird das MI-Prinzip „Develop Discrepancy“ umgesetzt [24];

- Methoden zum Umgang mit Widerstand;

- Methoden zur Förderung der Änderungszuversicht („Confidence Talk“). Diese Methoden dienen der Umsetzung des MIPrinzips „Support Self-Efficacy“.

\section{Aktuelle Projekte}

Die bisher beschriebenen Handlungsfelder und Prozesse der Frühinterventionen lassen sich größerenteils in einzelnen Projekten wiederfinden. Die nachfolgenden Tabellen geben eine Übersicht über aktuelle Projekte im Bereich Frühintervention. 
Zuerst werden Projekte vorgestellt, die sich mit Methoden der Frühintervention direkt an Betroffene wenden (Tab. 2). In einer weiteren Tabelle werden nachfolgend Projekte genannt, die sich nicht direkt an die Betroffenen, sondern an Lehrer, Ärzte, Erzieher etc. wenden (in Form von Peereducation und Multiplikatorenschulung), damit sie die Techniken der Früherkennung und der Frühintervention erlernen und anwenden können (Tab.3). Abschließend zeigt die letzte Tabelle Frühinterventionen, die speziell auf das Internet zugreifen und somit eine bestimmte Gruppe der Internet-User als Zielgruppe ansprechen (Tab. 4).

Neben den hier aufgelisteten Projekten im Bereich der Frühinterventionen ist der Suchtforschungsverbund Nord-Ost EARLINT zu nennen, einer der vier Forschungsverbünde für Suchtforschung, die vom Bundesministerium für Bildung und Forschung gefördert werden. EARLINT (Research Collaboration in Early Sub-

Tab. 2 Auszug von Frühinterventionsprojekten im deutschsprachigen Raum

\begin{tabular}{|c|c|c|c|c|}
\hline Methode & Zielsetzung & Zielgruppe & Träger & Laufzeit \\
\hline \multicolumn{5}{|c|}{ FreD Frühintervention bei erstauffälligen Drogenkonsumenten } \\
\hline $\begin{array}{l}\text { - Intake-Gespräch (Motiva- } \\
\text { tional Interviewing) } \\
\text { - mehrgliedriges Kursange- } \\
\text { bot }\end{array}$ & $\begin{array}{l}\text { - Verhinderung eines miss- } \\
\text { bräuchlichen Drogenkon- } \\
\text { sums mit erneuter straf- } \\
\text { rechtlicher Auffälligkeit }\end{array}$ & $\begin{array}{l}\text { - strafrechtlich auffällige Ju- } \\
\text { gendliche, heranwachsende } \\
\text { und junge Erwachsene }\end{array}$ & $\begin{array}{l}\text { Landschaftsverband West- } \\
\text { falen-Lippe, Koordinations- } \\
\text { stelle Sucht, Münster }\end{array}$ & $\begin{array}{l}\text { Pilotphase Oktober } 2000 \\
\text { bis Dezember } 2002 \\
\text { (evaluiert) }\end{array}$ \\
\hline \multicolumn{5}{|l|}{ Just be smokefree } \\
\hline $\begin{array}{l}\text { - Informationsbroschüre für } \\
\text { jugendliche Raucher mit } \\
\text { Hinweisen über das Rau- } \\
\text { chen } \\
\text { - zielgruppenspezifische } \\
\text { Homepage } \\
\text { - Unterstützung durch Multi- } \\
\text { plikatoren }\end{array}$ & $\begin{array}{l}\text { - Unterstützung für Jugend- } \\
\text { liche, die mit dem Rauchen } \\
\text { aufhören wollen } \\
\text { - rauchende Jugendliche } \\
\text { motivieren, sich mit ihren } \\
\text { Rauchgewohnheiten aus- } \\
\text { einander zu setzen }\end{array}$ & $\begin{array}{l}\text { - Jugendliche ab } 14 \text { Jahren } \\
\text { und junge Erwachsene bis } \\
\text { ca. } 25 \text { Jahren }\end{array}$ & $\begin{array}{l}\text { IFT-Nord, Institut für Therapie } \\
\text { und Gesundheitsforschung, } \\
\text { Kiel }\end{array}$ & $\begin{array}{l}\text { April bis Dezember } 2002 \\
\text { (evaluiert) }\end{array}$ \\
\hline \multicolumn{5}{|l|}{ EasyContact } \\
\hline $\begin{array}{l}\text { - frühzeitige Intervention bei } \\
\text { jungen Menschen mit } \\
\text { Suchtproblemen } \\
\text { - Erschließung von privaten } \\
\text { und institutionellen Res- } \\
\text { sourcen } \\
\text { - (Einzel-)Betreuung }\end{array}$ & $\begin{array}{l}\text { - Stabilisierung auf vorhande- } \\
\text { nem Niveau, Verhinderung } \\
\text { des weiteren Abstiegs } \\
\text { - Unterstützung des Ausstie- } \\
\text { ges aus dem Konsum von } \\
\text { Suchtmitteln, Orientierung } \\
\text { zum „cleanen“ Leben }\end{array}$ & $\begin{array}{l}\text { - minderjährige Jugendliche } \\
\text { bis } 21 \text { Jahren mit Suchtmit- } \\
\text { telproblemen }\end{array}$ & $\begin{array}{l}\text { Condrobs e. V. } \\
\text { München }\end{array}$ & fortlaufend \\
\hline \multicolumn{5}{|c|}{ Move - Motivierende Kurzintervention } \\
\hline $\begin{array}{l}\text { - dreitägige Schulungen mit } \\
\text { Multiplikatoren der Jugend- } \\
\text { hilfe aus Schulen, Vereinen, } \\
\text { Jungendzentren etc. } \\
\text { - Erlernen der Gesprächsfüh- } \\
\text { rung einer Kurzintervention } \\
\text { der MI (angepasst für Ju- } \\
\text { gendliche und ambulanten } \\
\text { Einsatz) }\end{array}$ & $\begin{array}{l}\text { - Initiierung einer Verhaltens- } \\
\text { änderung bei konsumieren- } \\
\text { den Jugendlichen } \\
\text { - Schließen der Versorgungs- } \\
\text { lücke für riskant konsumie- } \\
\text { rende Jugendliche } \\
\text { - Verbesserung der Koope- } \\
\text { ration zwischen Jugendhilfe } \\
\text { und Drogenhilfe }\end{array}$ & $\begin{array}{l}\text { - Jugendliche mit riskantem } \\
\text { Suchtmittelkonsum } \\
\text { - Personen, die mit diesen Ju- } \\
\text { gendlichen Kontakt haben } \\
\text { (z. B. Schulen, Vereine, Ju- } \\
\text { gendzentren, Einrichtungen } \\
\text { der Jugendhilfe) }\end{array}$ & $\begin{array}{l}\text { Ginko - Verein für psycho- } \\
\text { soziale Betreuung im Deut- } \\
\text { schen Paritätischen Wohl- } \\
\text { fahrtsverband, Landeskoordi- } \\
\text { nierungs-Stelle für } \\
\text { Suchtvorbeugung, Nordrhein- } \\
\text { Westfalen }\end{array}$ & $\begin{array}{l}\text { Projektbeginn Mai } 2000 \\
\text { (evaluiert) }\end{array}$ \\
\hline \multicolumn{5}{|c|}{ supra-f: Suchtpräventionsprogramm } \\
\hline $\begin{array}{l}\text { - zwölf supra-f-Zentren, Be- } \\
\text { treuungseinrichtungen in } \\
\text { der ganzen Schweiz } \\
\text { - Stützunterricht, Aufgaben- } \\
\text { hilfe, Sprachförderung, } \\
\text { Kommunikations- und Sozi- } \\
\text { altraining } \\
\text { - gemeinsames Mittagessen, } \\
\text { gemeinsame Freizeitgestal- } \\
\text { tung }\end{array}$ & $\begin{array}{l}\text { - Früherfassung von gefähr- } \\
\text { deten Jugendlichen } \\
\text { - Verbesserung der psy- } \\
\text { chischen Befindlichkeit } \\
\text { - Anbieten von Unterstüt- } \\
\text { zung, Selbstvertrauen ge- } \\
\text { winnen } \\
\text { - Einwirkung auf ihre Verhal- } \\
\text { tensweisen, die Probleme } \\
\text { verursachen } \\
\text { - Verminderung des Betäu- } \\
\text { bungsmittelkonsums }\end{array}$ & $\begin{array}{l}\text { - Unterstützung für Jugend- } \\
\text { liche in einer Risikosituation }\end{array}$ & $\begin{array}{l}\text { Bundesamt für Gesundheit, } \\
\text { BAG, Schweiz } \\
\text { Universität Fribourg }\end{array}$ & $\begin{array}{l}\text { 1999-2003, zweites Impuls- } \\
\text { programm (evaluiert) }\end{array}$ \\
\hline \multicolumn{5}{|l|}{ Hot mixes } \\
\hline $\begin{array}{l}\text { - konsum-, problem- und } \\
\text { zielgruppenspezifische } \\
\text { Präventionskampagne } \\
\text { zwei Plakate (Motiv Boy/ } \\
\text { Girl) } \\
\text { - Verteilung von Info-cards } \\
\text { mit gleichem Motiv bei } \\
\text { Großveranstaltungen (Goa } \\
\text { und Technoszene) durch } \\
\text { geschulte Peers }\end{array}$ & $\begin{array}{l}\text { - aufmerksam machen auf die } \\
\text { Gefahren des Drogenkon- } \\
\text { sums } \\
\text { - Bewusstsein schaffen für die } \\
\text { hohe gesundheitliche Ge- } \\
\text { fährdung bei Mischkonsum }\end{array}$ & $\begin{array}{l}\text { - Jugendliche und junge Er- } \\
\text { wachsene mit legalem und } \\
\text { illegalem Rauschmittelkon- } \\
\text { sum } \\
\text { - Teilnehmer von regional } \\
\text { bekannten Großveranstal- } \\
\text { tungen }\end{array}$ & $\begin{array}{l}\text { Büro für Suchtprävention, } \\
\text { BfS, Hamburg }\end{array}$ & Frühjahr 2001 (evaluiert) \\
\hline
\end{tabular}


Tab. 2 Fortsetzung, Auszug von Frühinterventionsprojekten im deutschsprachigen Raum

\begin{tabular}{|c|c|c|c|c|}
\hline Methode & Zielsetzung & Zielgruppe & Träger & Laufzeit \\
\hline \multicolumn{5}{|l|}{ Bekifft in der Schule } \\
\hline $\begin{array}{l}\text { - Bereitstellung einer Präven- } \\
\text { tionsfachkraft an jeder } \\
\text { Schule } \\
\text { - Bildung einer schulungs- } \\
\text { internen Steuerungs- und } \\
\text { Koordinationsgruppe } \\
\text { - Überprüfen des Umgangs } \\
\text { mit Cannabis }\end{array}$ & $\begin{array}{l}\text { - Problemfrüherkennung } \\
\text { Erlernen des Umgangs mit } \\
\text { Cannabis konsumierenden } \\
\text { Jugendlichen } \\
\text { - Unterstützung der Jugend- } \\
\text { lichen, ihren problemati- } \\
\text { schen Cannabiskonsum zu } \\
\text { erkennen und anzugehen }\end{array}$ & $\begin{array}{l}\text { - Schüler } \\
\text { - Lehrkräfte } \\
\text { - Eltern }\end{array}$ & $\begin{array}{l}\text { Sucht-Präventions-Zentrum, } \\
\text { Hamburg }\end{array}$ & $\begin{array}{l}\text { 2001-2003, } \\
\text { zweiter Durchlauf } 2003 \\
\text { (evaluiert) }\end{array}$ \\
\hline \multicolumn{5}{|c|}{ Mach den Test } \\
\hline $\begin{array}{l}\text { - Adaption des amerikani- } \\
\text { schen Manuals „Brief Alco- } \\
\text { hol Screening and Interven- } \\
\text { tion für College Students } \\
\text { (BASICS)“ } \\
\text { - Alters- und zielgruppen- } \\
\text { adäquate Intervention mit- } \\
\text { tels Broschüre } \\
\text { - Intervention erfolgt bei Be- } \\
\text { darf durch einstündiges } \\
\text { Feedbackgespräch, mit } \\
\text { Techniken des Motivational } \\
\text { Interviewing }\end{array}$ & $\begin{array}{l}\text { - Sensibilisierung hinsichtlich } \\
\text { Alkoholkonsum } \\
\text { - Vermeidung riskanter Trink- } \\
\text { muster (binge-drinking) } \\
\text { - Förderung eines verantwor- } \\
\text { tungsvollen Umgangs mit } \\
\text { Alkohol }\end{array}$ & $\begin{array}{l}\text { - Studenten aller Fachrichtun- } \\
\text { gen und Fakultäten }\end{array}$ & $\begin{array}{l}\text { IFT-Nord, Institut für Therapie } \\
\text { und Gesundheitsforschung, } \\
\text { Kiel }\end{array}$ & einmalig 2002 (evaluiert) \\
\hline
\end{tabular}

\section{Problem- und störungsorientierte Frühintervention bei Alkohol-, Cannabis- und Club-Drug-Konsumenten:}

Identifikation kognitiver und psychosozialer Problemprofile ventionen mitzuarbeiten

- Einstellung zur Reduzierung des Drogenkonsums, basierend auf der Grundlage von motivierender Gesprächsführung

\section{Fil rouge}

- Fortbildung in Prävention Sensibilisierung für die Früherkennung

- Entwicklung von Präventionskonzepten

\section{Next Step}

- Erlebnis- und Erfahrungskurse für gefährdete Jugendliche

- Beratung für Eltern, Angehörige und Lehrer

- Supervision und Organisationsentwicklung

\section{Pausezyt}

- niedrigschwellige Kontaktaufnahme mit erwachsener Person des Vertrauens

- Aufzeigen von Lösungsund Handlungsansätzen für den gefährdeten Schüler

\section{Spaß am Leben}

- Kursangebot für cannabiskonsumierende Jugendliche

- vier Abende durchgeführt von der Jugendberatungsstelle in Zusammenarbeit mit zwei Peers

- Empowerment-Strategie durch die Einbettung der zwei Jugendlichen als CoLeitung
- Untersuchung der Bereit- willigkeit, um bei Kurzinter-
- Identifikation kognitiver und - Teilgruppe der EDSP-Pro- psychosozialer Problempro- banden
Suchtforschungsverbund Bayern/Dresden, ASAT
1.7.2002-1.2.2005 (evaluiert) file
- Verankerung von Gesundheitsförderung und Prävention in 35 Kinder- und Jugendheimen und in der Heimstruktur

- Sicherstellung eines Beratungs- und Betreuungsangebotes für gefährdete Schüler

- Erarbeitung eines schulinternen Handlungsmodells für den Fall des Suchtmittelkonsums
- frühzeitiges Erkennen gefährdeter Schüler Fernhalten von individuellen bzw. Gruppenproblemen

- Entlastung der Lehrkräfte vom Schulbetrieb

\section{- Bewohner von Kinder- und} Jugendheimen, Mitarbeiter und Leitende von Heimen

- Lehrkräfte, Schulleitung

- Lehrkräfte, Schulleitung V Vivid - Fachstelle für Suchtund deren Bezugspersonen
Bundesamt für Gesundheit, Hochschule für soziale Arbeit,

Luzern,

Schweiz prävention, Graz, Österreich

März 2002 bis Dezember 2004 (evaluiert)
- Auseinandersetzung mit dem Cannabiskonsum

- Reflexion von Genussalternativen
- Oberstufenschüler

- Lehrkräfte

Jugendliche im Alter von 16-20 Jahren offene Jugendarbeit, Jugendtreff Weinfeld, Bürglen, Schweiz 2003, ab dann Eingliederung in offene Jugendarbeit
Herbst 2000 bis Sommer

Jugend-, Eltern- und Sucht- andauernd beratungsstelle Contact, Bern 
Tab. 2 Fortsetzung, Auszug von Frühinterventionsprojekten im deutschsprachigen Raum

\begin{tabular}{|c|c|c|c|c|}
\hline Methode & Zielsetzung & Zielgruppe & Träger & Laufzeit \\
\hline \multicolumn{5}{|c|}{ Junge Männer im Straßenverkehr - Voll im Griff } \\
\hline $\begin{array}{l}\text { - Workshop „Alkohol trinken } \\
\text { und Auto fahren“ } \\
\text { - Autofahren auf einem Par- } \\
\text { cours unter Alkoholkon- } \\
\text { sum, Videoaufzeichnung } \\
\text { der Fahrt } \\
\text { - Auswertung der Videos in } \\
\text { Hinblick auf Veränderungen } \\
\text { des Fahrverhaltens } \\
\text { - suchtmittelspezifische Auf- } \\
\text { klärung }\end{array}$ & $\begin{array}{l}\text { - Vermeidung von Fahren } \\
\text { unter Alkoholeinfluss } \\
\text { - Senkung der Inzidenz der } \\
\text { alkoholbedingten Unfälle in } \\
\text { der Zielgruppe } \\
\text { - Förderung der Punktnüch- } \\
\text { ternheit }\end{array}$ & $\begin{array}{l}\text { - junge Männer zwischen } 18 \\
\text { und } 25 \text { Jahren }\end{array}$ & $\begin{array}{l}\text { Niedersächsische Landesstelle } \\
\text { gegen die Suchtgefahren, } \\
\text { Hannover }\end{array}$ & $1998-2000$ (evaluiert) \\
\hline
\end{tabular}

Tab. 3 Auszug von Weiterbildungs- und Schulungsangeboten

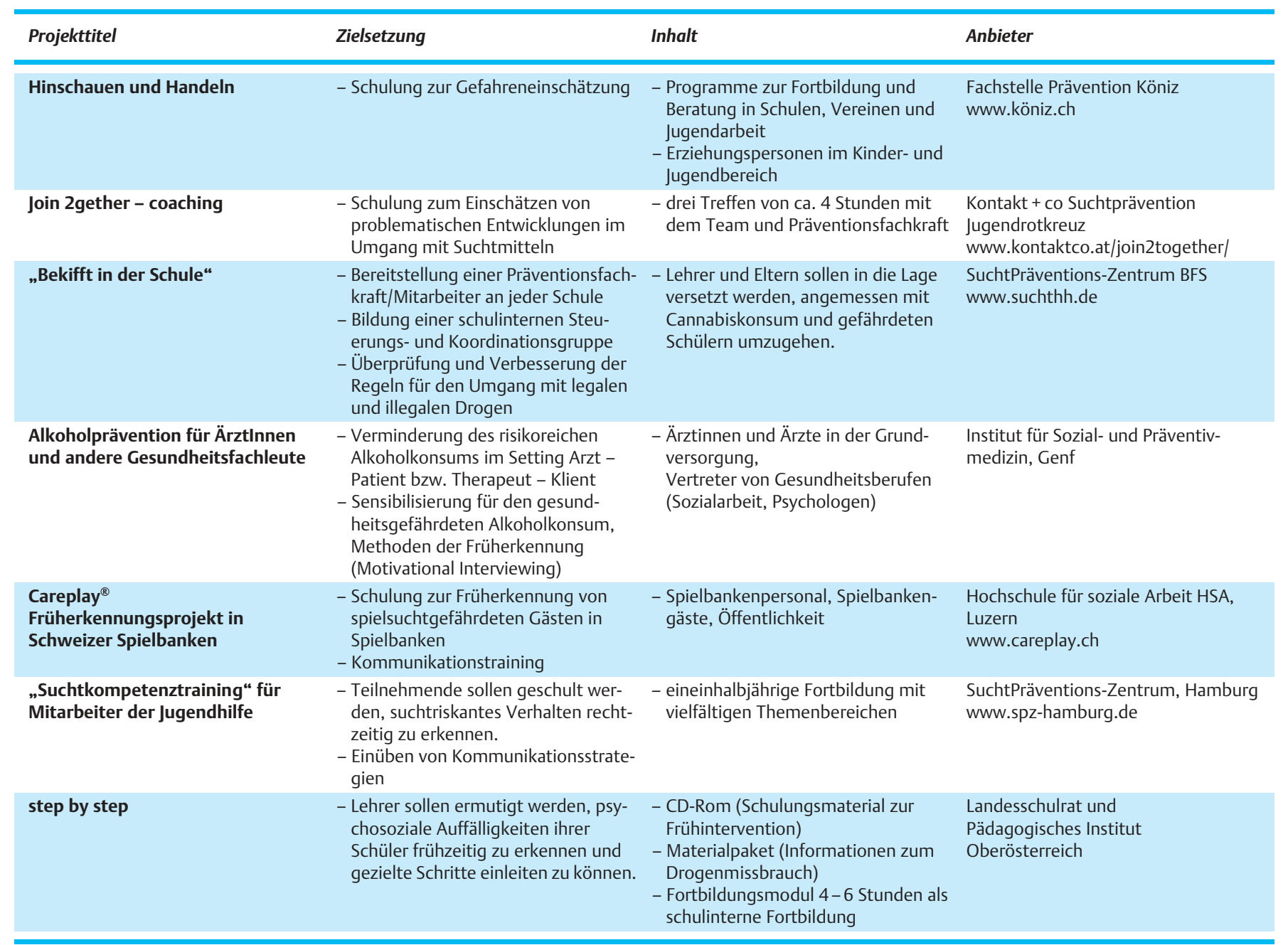

stance Use Intervention) entwickelt Kurzinterventionsansätze bezogen auf alkohol- oder tabakrauchassoziierte Störungen. Durchführungsorte sind hier Einrichtungen der medizinischen Versorgung sowie Einrichtungen der „Allgemeinbevölkerung“. Das Hauptziel dieses Forschungsverbundes ist somit eine Verbesserung der Mängel im Bereich Früh- bzw. Primärprävention substanzbezogener Störungen. EARLINT umfasst zurzeit sechs
Forschungsprojekte, die alle eine Wirksamkeitsprüfung von Interventionen mittels experimenteller Felduntersuchungen beinhalten. Als theoretische Grundlage der von EARLINT durchgeführten und evaluierten Interventionen dient das Transtheoretische Modell der Verhaltensänderung, als Interventionsform wird die motivierende Gesprächsführung gewählt [26]. So hat beispielsweise ein Projekt das Ziel, die Effektivität und den Im- 
Tab. 4 Auszug von Frühinterventionsprojekten für Internet-User

\begin{tabular}{|c|c|c|c|c|}
\hline Methode & Zielsetzung & Zielgruppe & Träger & Laufzeit \\
\hline \multicolumn{5}{|c|}{ Drugcom.de (www.drugcom.de) } \\
\hline $\begin{array}{l}\text { - interaktives Informations- } \\
\text { und Kommunikationsportal } \\
\text { - Informationsmöglichkeit } \\
\text { über legale und illegale } \\
\text { Drogen } \\
\text { - persönliche Beratung }\end{array}$ & $\begin{array}{l}\text { - Anregung der Kommunika- } \\
\text { tion über Drogen und Sucht } \\
\text { - selbstkritische Auseinander- } \\
\text { setzung mit dem eigenen } \\
\text { Konsumverhalten } \\
\text { - Förderung eines risikoarmen } \\
\text { Umgangs mit psychoaktiven } \\
\text { Substanzen }\end{array}$ & $\begin{array}{l}\text { - Jugendliche zwischen } 15 \\
\text { und } 22 \text { Jahren }\end{array}$ & $\begin{array}{l}\text { Bundeszentrale für gesund- } \\
\text { heitliche Aufklärung, BzgA, } \\
\text { Köln }\end{array}$ & seit 2001 \\
\hline \multicolumn{5}{|l|}{ feelok (www.feelok.ch) } \\
\hline $\begin{array}{l}\text { - wissenschaftlich fundiertes } \\
\text { internetbasiertes Interven- } \\
\text { tionsprogramm zur Canna- } \\
\text { bis-, Stress- und Tabakprä- } \\
\text { vention } \\
\text { - Anschauungsmaterial für } \\
\text { den Unterricht } \\
\text { - stadienspezifische Inter- } \\
\text { vention auf Grundlage des } \\
\text { Transtheoretischen Modells }\end{array}$ & $\begin{array}{l}\text { - motiviert Jugendliche, } \\
\text { Nichtraucher zu bleiben } \\
\text { - rauchende Jugendliche zu } \\
\text { motivieren, weniger zu rau- } \\
\text { chen oder das Rauchen ein- } \\
\text { zustellen } \\
\text { - Unterstützung im Entwöh- } \\
\text { nungsprozess }\end{array}$ & $\begin{array}{l}\text { - Jugendliche zwischen } 10 \\
\text { und } 18 \text { Jahren }\end{array}$ & $\begin{array}{l}\text { Institut für Sozial- und } \\
\text { Präventivmedizin, Zürich }\end{array}$ & $\begin{array}{l}\text { Entwicklung und Evaluation } \\
\text { seit } 1999, \text { Implementierung } \\
\text { seit } 2003 \\
\text { (evaluiert) }\end{array}$ \\
\hline
\end{tabular}

pact von Strategien der Raucherentwöhnung unter Allgemeinarztpraxisbedingungen zu evaluieren, die eine hohe Gewähr für eine Implementierung in der ärztlichen Routinebehandlung aufweisen.

\section{Fazit}

Die Erkenntnis, dass Menschen mit missbräuchlichem Drogenkonsum häufig erst zu spät erreicht werden, meist zu einem Zeitpunkt, wo bereits massive Folgekrankheiten und hohe Folgekosten bestehen, zeigt die Notwendigkeit des frühzeitigen Handelns. Frühinterventionen sind ein geeignetes Instrument, um zielgruppenorientiert Personen mit missbräuchlichem oder riskantem Konsumverhalten frühzeitig anzusprechen, hierzu sind Zugangswege und Aktionsfelder auch außerhalb des klassischen Suchthilfesystems zu suchen.

Die Praxis zeigt bislang, dass es bei der Umsetzung bzw. bei der Anwendung von Frühinterventionen in Deutschland an geeigneten Rahmenbedingungen mangelt wie z. B. die nicht bestehenden Abrechnungsmöglichkeiten für die Erbringung von Frühinterventionen in der Arztpraxis und im Krankenhaus. Es fehlt eine Diagnose, die den „riskanten Konsum, jedoch ohne Abhängigkeitskriterien“ beschreibt und somit Frühinterventionen als suchtspezifische Leistungen anerkennt und honoriert. Als eine weitere Optimierung der Versorgung von Personen mit riskantem Substanzkonsum oder mit erhöhtem Abhängigkeitsrisiko sind strukturelle Voraussetzungen zu nennen. Hierzu zählen die Vernetzung und Kooperation der Akteure aus dem Suchtkrankenhilfesystem und dem Bereich der medizinischen Basisversorgung sowie verschiedener Akteure aus anderen Handlungsfeldern von Frühinterventionen. Auch wenn inzwischen ein breiter Konsens über die Notwendigkeit des frühzeitigen Handelns besteht und dass dies durch geeignete Rahmenbedingungen zu verbessern wäre, werden diese Erkenntnisse noch nicht zufrieden stellend umgesetzt. Hier sind gesundheitspolitische Handlungen erforderlich.

Grundlage für eine relevante Verbreitung von Frühinterventionen sind ähnlich wie bei der Prävention ihre Gewichtung unter den Beschäftigten sowohl des Suchthilfesystems als auch des medizinischen Systems, das die Masse der Suchtkranken zumindest begleitet. Die Aufgabenteilung könnte in der Entwicklung und Bereitstellung geeigneter und niedrigschwelliger Interventionen seitens der Suchtfachleute und einer Integration in das Hausarzt- und Allgemeinmedizinsystem seitens der Grundversorgung sein. Vielleicht gibt die Förderung der integrierten Versorgung durch das neue GMG hier Anstöße. Auch die Schule oder die Verkehrserziehung sind als Ort der Frühintervention gut geeignet, wenn sie getragen sind von einem Problembewusstsein der entsprechenden Systeme. Hier besteht eine Gemeinsamkeit mit der Prävention. Eine öffentliche Diskussion zur Antistigmatisierung Suchtkranker, wie die der Deutschen Gesellschaft für Suchtmedizin, ist hier ein gutes Beispiel; sie wendet sich gegen Diskriminierung und gegen das Versagen von Hilfe.

Eine hohe Relevanz ist dem Forschungsbedarf von Studien und Projekten in dem relativ jungen Gebiet der Frühinterventionen zuzusprechen, denn nur so lässt sich klären, bei welcher spezifischen Zielgruppe und bei welcher Substanz Frühinterventionen wirksam sind.

\section{Literatur}

${ }^{1}$ Die Drogenbeauftragte der Bundesregierung, Bundesministerium für Gesundheit und Soziale Sicherheit. Aktionsplan Drogen und Sucht. November 2003

${ }^{2}$ Rumpf HJ, Bischof G, Fritze M et al. Aktionsplan Alkohol SchleswigHolstein: Frühentdeckung und Kurzintervention bei riskantem Alkoholkonsum in der medizinischen Versorgung. Schleswig-Holsteinisches Ärzteblatt 2003; 9: $67-73$ 
${ }^{3}$ Die Drogenbeauftragte der Bundesregierung, Bundesministerium für Gesundheit und Soziale Sicherheit. Drogen- und Suchtbericht. April 2003

${ }^{4}$ Ezzati M, Lopez AD, Rodgers A et al., and the Comparative Risk Assessment Collaborating Group.. Selected major risk factors and global and regional burden of disease. Lancet 2002; 360: 1347 - 1360

5 World Health Organization. World Report on Road Traffic Injury Prevention. Geneva 2004

${ }^{6}$ Siegmund S, Teyssen S, Singer MV. Alkoholassoziierte Organschäden. Internist 2002; 43: 287-293

${ }^{7}$ Bühringer G, Augustin R, Bergmann E et al. Alkoholkonsum und alkoholbezogene Störungen in Deutschland. Baden-Baden: Nomos,, 2000

${ }^{8}$ Rehm J. Suchtmittel und Public Health. Suchttherapie 2003; 4: 72 - 75

${ }^{9}$ Klein M. Suchtstörungen [Addictive disorders]. In: Brinkmann-Göbel $\mathrm{R}$ (Hrsg). Handbuch für Gesundheitsberater. Bern: Huber, 2001

${ }^{10}$ Rumpf HJ, Meyer C, Hapke U et al. Inanspruchnahme suchtspezifischer Hilfen von Alkoholabhängigkeits und -mißbrauchern: Ergebnisse der TACOS Bevölkerungsstudie. Sucht 2000; 46: 9-17

${ }^{11}$ Bundeszentrale für gesundheitliche Aufklärung (BZgA). Kurzintervention bei Patienten mit Alkoholproblemen, Köln: BzgA, 2001

${ }^{12}$ Rilke O. Alkoholbezogenen Störungen in Sachsen, Epidemiologie und Möglichkeiten früher Interventionen. Ärzteblatt Sachsen 2004; 2: $52-55$

13 John U, Hapke U, Rumpf HJ et al. Prävalenz und Sekundärprävention von Alkoholmissbrauch und- abhängigkeit in der medizinischen Versorgung. Baden-Baden: Nomos, 1996

${ }^{14}$ Rumpf HJ, Hapke U, John U. Der Lübecker Alkoholabhängigkeits- und -missbrauchs-Screnning-Test (LAST). Manual. Göttingen: Hogrefe Verlag für Psychologie, 2001

${ }^{15}$ Mayfield D, McLeod G, Hall P. The CAGE questionnaire: validation of a new alcoholism screening instrument. Am J Psychiatry 1974; 131 (10): $1121-1123$
${ }^{16}$ Feuerlein W, Küfner H, Ringer C et al. Münchner Alkoholismus Test (MALT). Weinheim: Beltz, 1977

17 Saunders JB, Aasland OG, Babor TF et al. Development of the Alcohol Use Disorders Screening Test (AUDIT). WHO collaborative project on early detection of persons with harmful alcohol consumption. Addiction 1993; 88: $791-804$

${ }^{18}$ Kremer G. Frühintervention und Kurzintervention - ein neues Heilmittel? In: Frühintervention bei erstauffälligen Drogenkonsumenten. Münster: Landschaftsverband Westfalen-Lippe, 2003

${ }^{19}$ Miller WR, Sanchez VC. Motivating your adults for treatment and lifestyle change. In: Howard GS, Nathan PE (Hrsg). Alcohol use and misuse by young adults. Notre Dame: University of Notre Dame Press, 1994

${ }^{20}$ Velicer WR, Prochaska JO, Fava JL et al. Smoking cessatin and stress management: Application of the Transtheoretical Model of behavior change. Homestasis 1998; 38: 216-233

${ }^{21}$ Maurischat C. Erfassung der „Stages of Change“ im Transtheoretischen Modell Prochaska's - eine Bestandsaufnahme. Forschungsberichte des Psychologischen Instituts der Albert-Ludwigs-Universität Freiburg i.Br., Nr. 154, 2001

${ }^{22}$ Prochaska JO, Velicier WF, Rossi JS et al. Stages of Change and Decisional Balance for 12 Problem Behaviors. Health Psychology 1994; 13: $39-46$

${ }^{23}$ Rollnick S, Miller WR. What is motivational interviewing? Behavioural and Cognitive Psychotherapy 1995; 23: $325-334$

${ }^{24}$ Körkel J, Veltrup C. Motivational Interviewing: Eine Übersicht. Suchttherapie 2003; 4: 115-124

${ }^{25}$ Miller WR, Rollnick S. Motivational Interviewing. Preparing People for change. New York: Guilford, 2002

${ }^{26}$ Mann K, Gastpar M, John U et al. Forschungsverbünde für Suchtforschung: Fragestellungen und Inhalte. Sucht Aktuell 2003; 1: 50-56 